\title{
Characterisation of microsatellite loci in two species of lice, Polyplax serrata (Phthiraptera: Anoplura: Polyplacidae) and Myrsidea nesomimi (Phthiraptera: Amblycera: Menoponidae)
}

\author{
Jana Martinů ${ }^{1,2}$, Veronika Roubová ${ }^{1}$, Milena Nováková ${ }^{1}$, Vincent S. Smith ${ }^{3}$, Václav Hypša ${ }^{1,2}$ and Jan Štefka ${ }^{1,2}$ \\ ${ }^{1}$ Faculty of Science, University of South Bohemia, České Budějovice, Czech Republic; \\ ${ }^{2}$ Institute of Parasitology, Biology Centre of the Czech Academy of Science, České Budějovice, Czech Republic; \\ ${ }^{3}$ Life Sciences Department, Natural History Museum, London, United Kingdom
}

\begin{abstract}
Polymorphic microsatellite loci were characterised for two louse species, the anopluran Polyplax serrata Burmeister, 1839, parasitising Eurasian field mice of the genus Apodemus Kaup, and the amblyceran Myrsidea nesomimi Palma et Price, 2010, found on mocking birds endemic to the Galápagos Islands. Evolutionary histories of the two parasites show complex patterns influenced both by their geographic distribution and through coevolution with their respective hosts, which renders them prospective evolutionary models. In P. serrata, 16 polymorphic loci were characterised and screened across 72 individuals from four European populations that belong to two sympatric mitochondrial lineages differing in their breadth of host-specificity. In M. nesomimi, 66 individuals from three island populations and two host species were genotyped for 15 polymorphic loci. The observed heterozygosity varied from 0.05 to 0.9 in P. serrata and from 0.0 to 0.96 in M. nesomimi. Deviations from the Hardy-Weinberg equilibrium were frequently observed in the populations of both parasites. Fst distances between tested populations correspond with previous phylogenetic data, suggesting the microsatellite loci are an informative resource for ecological and evolutionary studies of the two parasites.
\end{abstract}

Keywords: ectoparasite, population genetics, coevolution, Polyplax, Myrsidea, evolution, Europe, Galápagos

Parasitic lice (Phthiraptera) represent long-standing models for the study of host-parasite co-speciation. Many species of sucking lice (Anoplura) and two chewing louse suborders (Amblycera and Ischnocera) have been scrutinised with molecular methods to reveal complex evolutionary histories. These range from strict co-speciation to completely decoupled host-parasite phylogenies (e.g. Johnson and Clayton 2004, Demastes et al. 2012, Mizukoshi et al. 2012) and offer a unique opportunity to study the microevolutionary processes associated with the parasite's dependence on its host and geographic distribution.

Multilocus markers are a critical source of population genetic data necessary to tease apart the different patterns generated by these processes. With the exception of human louse (Leo et al. 2005, Ascunce et al. 2013), such data are still scarce and little is known about the genetic background of louse populations. Recent microsatellite studies include work on Degeeriella regalis Giebel, 1866, an ischnoceran louse parasitising Galapagos hawks (Koop et al. 2014) and preliminary data on two additional chewing louse species, Colpocephalum turbinatum Denny, 1842 and Geomydoecus ewingi Price et Emerson, 1971 (Peters et al. 2009, Nessner et al. 2014). In the present study, we extend this set by characterising polymorphic microsatellite loci in two more louse species, the anopluran Polyplax serrata Burmeister, 1839 and the amblyceran Myrsidea nesomimi Palma et Price, 2010. These lice have been the subjects of recent co-evolutionary research (Štefka and Hypša 2008, Štefka et al. 2011) and have the potential to serve as a model for microevolutionary studies of host-parasite interactions.

Polyplax serrata belongs to a cosmopolitan family Polyplacidae parasitising rodents (Light et al. 2010). It is found almost exclusively on the field mice genus Apodemus Kaup, and exceptionally on some other rodent taxa such as Clethrionomys glareolus (Schreber) or Microtus arvalis (Pallas) - Krištofík and Lysý (1992). Polyplax serrata, together with its hosts, occurs in the whole of Eurasia and has a complex population-genetic pattern that is partly influenced by geographical structure and partly by the host. According to Štefka and Hypša (2008), P. serrata is composed of three mitochondrial lineages, one parasitising two host species, Apodemus agrarius (Pallas) and A. uralensis (Pallas) (lineage $\mathrm{C}$ ), and two sympatric lineages that differ in the extent of their host specificity: lineage A lives on two host species, A. flavicollis (Melchior)

Address for correspondence: J. Martinů, Institute of Parasitology, Biology Centre of the Czech Academy of Science, Branišovská 31 , 370 05 České Budějovice, Czech Republic. Phone: (+420) 387775448; Fax: (+420) 385310388; E-mail: martinu@paru.cas.cz 
and $A$. sylvaticus (Linneaus), whereas lineage B possesses high affinity to A. flavicollis. Given that lice from the lineages $\mathrm{A}$ and $\mathrm{B}$ occur in sympatry throughout Europe and are composed of clearly distinct lineages, they likely represent cryptic species. However, the original reconstruction of their evolutionary history was based on short fragments of mitochondrial cox 1 and nuclear $E F-1 \alpha$ genes (Štefka and Hypša 2008). These provide reliable information on past isolation events, but may not be informative enough to indicate recent processes (e.g. post-glacial admixture of the lineages or recent fluctuations in population sizes).

The second model species, Myrsidea nesomimi, parasitises all four species of mockingbirds allopatrically distributed on the Galápagos islands (Mimus parvulus Gould, M. trifasciatus Gould, M. melanotis Gould and M. macdonaldi Ridgway). Studying mitochondrial DNA diversity of mockingbird lice from 11 islands, Štefka et al. (2011) found co-phylogeographic patterns shared by the louse and most of its host populations. The study, however, also revealed several incongruences. For example, despite a recent common origin of the neighbouring populations of an endangered Floreana mockingbird, surviving on the islets of Champion and Gardner by Floreana (Hoeck et al. 2010), their louse populations were not related. Instead, the $M$. nesomimi population from Champion showed a closer relationship to populations from Santa Fe in the central part of the archipelago.

For both model species, multilocus loci such as microsatellites could provide additional data critical for tracking recent migrations of these parasites and help to delineate the shape of the population structure in the parasite and its host. The present study focused on the development and characterisation of multiplex panels of genetically informative, polymorphic microsatellite loci (STR - short tandem repeats) in both species of lice. Such tools will help to extend our knowledge on the population genetics of parasites and detect historically recent events in the evolution of these two louse species.

\section{MATERIALS AND METHODS}

Two strategies were employed to obtain sequences of candidate microsatellite loci from the two louse genomes. First, the protocol of Fleischer and Loew (1995) was followed to construct the microsatellite-enriched libraries, clone them into E. coli plasmids and sequence them using Sanger sequencing. Due to a relatively low number of loci obtained in this way, a second strategy based on Next Generation Sequencing (NGS) was employed. Pooled extracts of louse DNA were used for constructing enriched libraries and sequenced with NGS technology (454, Roche, Brandford, USA) in a commercial laboratory (GenoScreen, Lille, France).

The protocol described by Fleischer and Loew (1995) was adopted with minor modifications (available upon request). Two oligonucleotides, comprising $13 \mathrm{CA}$ and 13 AT repeats, were used to produce GT and TA microsatellite-enriched libraries. Both oligos were biotin-labelled at the 5 'end and a three-carbon spacer was added to the 3'end (manufactured by Generi-Biotech, Hradec Králové, Czech Republic) to prevent the oligos from acting as a primer in the subsequent polymerase chain reaction (PCR) steps of the subtraction (Koblížková et al. 1998). Following this protocol, 120 and 68 microsatellite sequences were isolated for P. serrata and $M$. nesomimi, respectively.

Sixty and 24 sequences (for P. serrata and M. nesomimi, respectively), for which stable primer pairs could be designed, were selected for PCR testing. Structure of the microsatellite and surrounding regions were used as guidelines when selecting the candidates. The primers were constructed manually in PrimerSelect (DNASTAR, Madison, USA). The testing sample included populations representing two sympatric lineages, A and B, delimited by Štefka and Hypša (2008) for P. serrata and five Galápagos islands (Santa Cruz, Santa Fe, Española, Champion and San Cristobal) for M. nesomimi. One P. serrata individual per population (French isolate from lineage A, Slovakian isolate from eastern mitochondrial cluster of the lineage $\mathrm{B}$ and $\mathrm{Czech}$ isolate from western cluster of the lineage B) were used to test successful PCR amplification with the designed microsatellite primers.

The total volume of reaction was $10 \mu \mathrm{l}$, containing approximately $15 \mathrm{ng}$ of template DNA, $5 \mu \mathrm{l}$ of 2x QIAGEN Multiplex PCR Master Mix (QIAGEN Multiplex PCR Kit, Qiagen, Hilden, Germany), $2 \mu 1$ 5x Q-Solution, and $\mathrm{H}_{2} \mathrm{O}$. Forward primers were labelled with 6-FAM fluorescent dye at the 5'end (Sigma Aldrich, Praha, Czech Republic). The thermal profile for P. serrata was as follows: $3 \mathrm{~min}$ at $94^{\circ} \mathrm{C}$ followed by 29 cycles of $15 \mathrm{~s}$ at $94^{\circ} \mathrm{C}$, $30 \mathrm{~s}$ at $58^{\circ} \mathrm{C}$ and $1 \mathrm{~min}$ at $72^{\circ} \mathrm{C}$; the duration time of the final extension was $15 \mathrm{~min}$ at $72^{\circ} \mathrm{C}$. For M. nesomimi, the annealing temperature was set to $55^{\circ} \mathrm{C}$ and three individuals per each of the five islands were tested. Five microlitres of each PCR product were checked on a $2 \%$ agarose gel along with a 100 -bp marker. PCR products of loci that showed satisfactory amplification were diluted to $1: 10$ and $1: 100$ and analysed on the ABI 3100 genetic analyser (Applied Biosystems, Life Technologies Czech Republic s.r.o., Praha, Czech Republic). Using the PCR conditions described above, five and four of the loci were found to be polymorphic in at least one of the tested P. serrata and M. nesomimi populations, respectively (see Tables 1 and 2 for primer description).

For NGS technology and selection of additional candidate microsatellite loci, DNA pools were prepared from (a) a total of 15 individuals of P. serrata from A and B lineages, and (b) nine individuals of $M$. nesomimi from the Santa Cruz population. The genomic DNA was isolated using the QIAamp ${ }^{\circledR}$ DNA Micro Kit (QIAGEN). The concentration and purity of genomic DNA was verified spectrophotometrically by NanoDrop 3 (NanoDrop Technologies, Inc., Wilmington, Delaware, USA). Obtained DNA was sent to GenoScreen for commercial NGS service GenoSat ${ }^{\circledR}$. The NGS service analysis resulted in 455 microsatellite loci candidates in each species, containing di-, tri-, tetra-, penta-, and hexanucleotide repeats of length varying between 90-320 bp. Ninety loci containing various di- and trinucleotide repeat motifs were selected for tests of successful PCR amplification on P. serrata individuals from lineages A and $\mathrm{B}$, as well as 34 loci in $M$. nesomimi on individuals from 5 islands. Conditions of PCR reactions and visualisation of samples were the same as described above.

Out of 90 and 34 loci tested in PCR, 23 and 17 loci provided PCR product of the expected size in individuals of $P$. serrata and M. nesomimi, respectively. Louse extracts typically produce low DNA yields allowing a limited number of PCRs, thus different louse specimens were used to determine STR allele polymorphism for 454 obtained loci compared to the tests above. The 


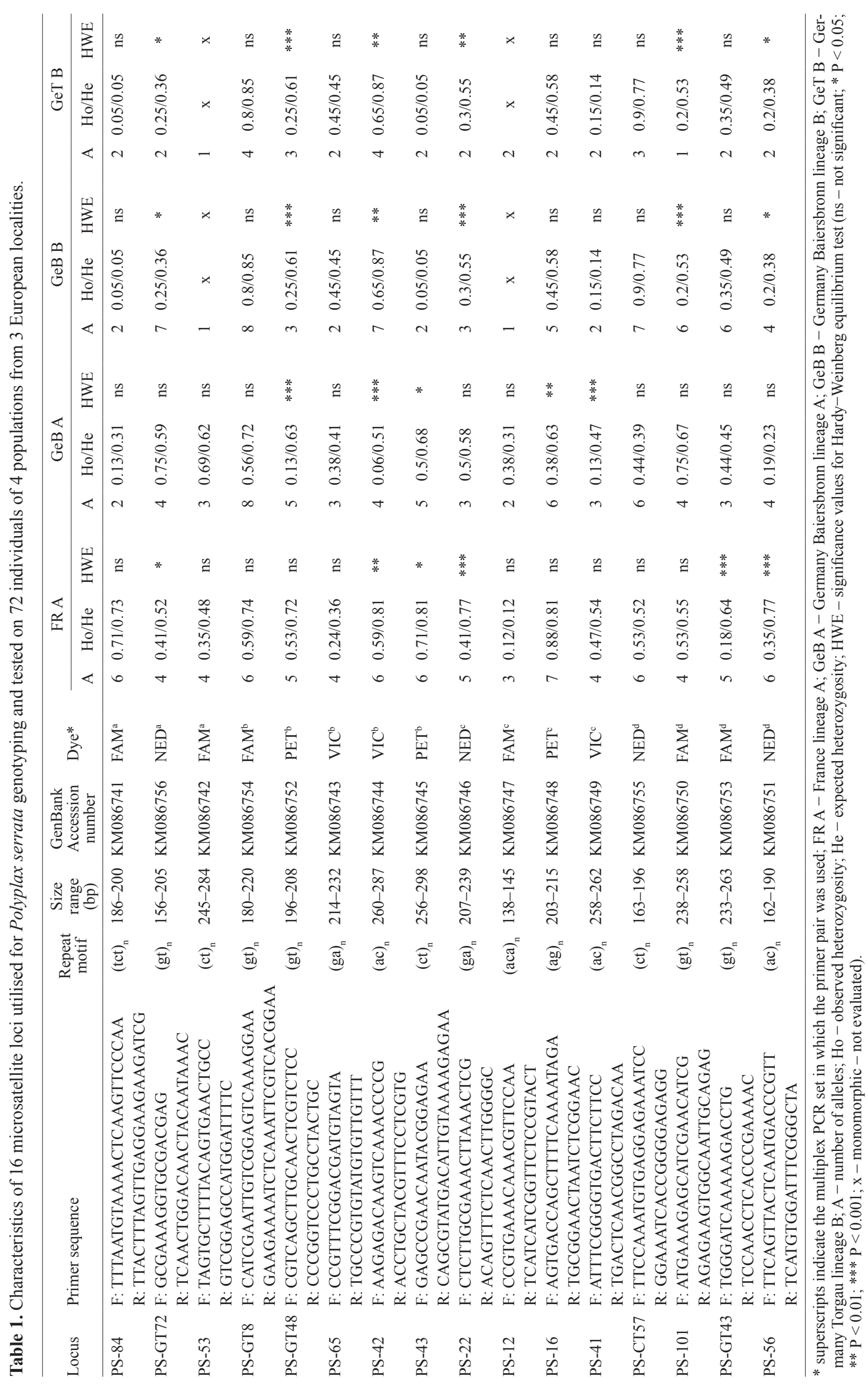




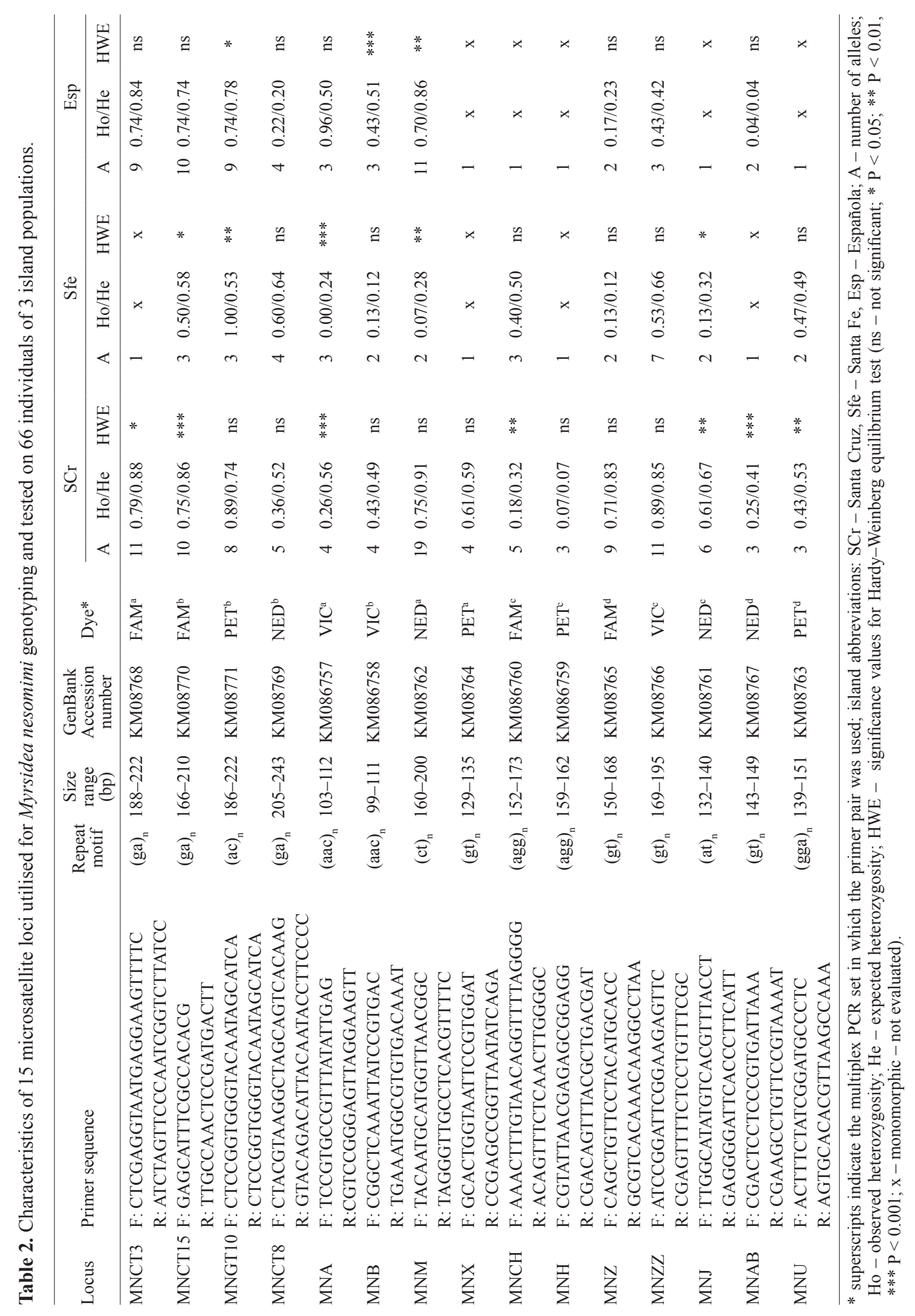


candidate loci were tested using a fragment analysis with fluorescently labelled primers for ten $P$. serrata individuals from one German locality (lineage B) and ten individuals from one French locality (lineage A). Out of these loci, 12 were excluded, either due to failure to amplify PCR product in several individuals (potential occurrence of null alleles), or because some loci were detected to be monomorphic. In M. nesomimi, candidate loci were tested using the same approach with ten individuals from the Santa Cruz and Española populations, respectively.

Finally, loci found to be polymorphic in at least one of the screened populations were subjected to the tests of Hardy-Weinberg equilibrium (HWE) and Linkage Disequilibrium (LD) using population sets of representative sizes. Eleven NGS obtained loci polymorphic in $P$. serrata (2-11 alleles per locus), together with five polymorphic loci gained from GT and TA libraries, were amplified in four multiplex PCR assays (the same conditions as for the STR loci polymorphism above). Similarly in M. nesomimi, four multiplex panels were created for 15 polymorphic loci (11 loci obtained from the NGS screening and four loci from the GA and TA libraries). Combinations of the markers used in the multiplexed assays are specified in Tables 1 and 2. For each panel, either different dyes were used for loci that overlapped in size, or a single dye was applied to PCR products of different sizes. PCR reactions were sent to the commercial service (Macrogen Inc. Korea and Netherlands, Seoul, Korea) for fragment analysis. The GeneMapper v.3.7 software (Applied Biosystems) was used for genotyping. In P. serrata, the 16 loci were tested for genotypic equilibrium and deviations from Hardy-Weinberg proportions. Seventy-two individuals coming from two localities from lineage $\mathrm{A}$ and two localities from lineage B (17 individuals from French lineage A, 15 from Baiersbronn German lineage A, 21 from Baiersbronn German lineage B and 19 from Torgau German lineage B) were analysed to evaluate performance of the markers (Table 1). In M. nesomimi, 66 individuals from three island populations (28 individuals from Santa Cruz, 15 from Santa Fe and 23 from Española) were used (Table 2). Tests for HWE and LD were performed online in the Genepop website (http://genepop.curtin.edu.au/). Fst values between all population pairs were calculated in Genalex 6.5 (Peakall and Smouse 2012), using 9999 permutations to test the significance of the results.

All microsatellites were also tested with extracts of pure host DNA to exclude possible cross-amplification with contaminant host DNA from a bloodmeal or skin and other host tissues in the louse gut.

\section{RESULTS AND DISCUSSION}

Levels of polymorphism detected in the tested populations (number of alleles, heterozygosity) and results of the HWE tests are listed in Tables 1 and 2. In P. serrata, all 16 loci were found to be polymorphic in populations from the lineage A (Germany Baiersbronn, France) with the number of alleles ranging from two to seven. Populations from lineage B (Germany Baiersbronn, Germany Torgau) were monomorphic in two loci (PS-12, PS-53). Significant deviations from HWE $(\mathrm{P}<0.05)$ were observed in all four populations: five loci deviated in Germany Baiersbronn (lineage A) and six loci in Germany Baiersbronn (lineage B), Germany Torgau (lineage B) and France (lineage A; Table 1) populations. Overall, four loci were in HWE in all test populations and only one locus (PS-42) deviated from
HWE across the studied set (Table 1). We therefore suggest that the PS-42 locus is excluded from further application.

All 16 loci were tested for LD, applying sequential Bonferroni correction for multiple comparisons. The tests were not significant for two populations, France lineage A and Germany Torgau lineage B. Out of the 120 locus-pair combinations tested, seven showed significant deviation in the Germany Baiersbronn lineage A (PS-GT72 and PS-53; PS42 and PS-41; PS-GT72 and PS-101; PS-53 and PS-101; PS-42 and PS-12; PS-12 and PS-41; PS-GT72 and PS-42) and six in the Baiersbronn lineage B (PS-GT43 and PS-16; PS-GT43 and PS-101; PS-16 and PS-56; PS-101 and PS56; PS-GT43 and PS-56; PS-16 and PS-101). Positive LD tests in the two populations may reflect a hidden genetic structure caused by using up to seven louse individuals per host individual (Koop et al. 2014). Louse populations inbreed for multiple generations on a single host individual, which may result in a sub-population with low diversity and genetic loci inherited in linked blocks. In the populations from France and Germany (Torgau), a maximum of two individuals per host were used, thus lessening this kind of artifact. Whilst deviation from HWE due to a reduced number of heterozygotes is still present, the level of LD in these populations is not significant. Fst pairwise distances among the four populations correspond to the mtDNA-based delimitation of the lineages: in all comparisons the within-lineage distances were lower ( Fst $=0.206$ and 0.230) than distances between the lineages, even for the two sympatric Baiersbronn populations (Fst $=0.283$ to $0.351)$. All Fst values were significant at $\mathrm{P} \leq 0.001$.

In M. nesomimi, all 15 loci were found polymorphic in the Santa Cruz population and eight loci were polymorphic across all three populations (Table 2). The levels of heterozygosity were low in many loci and deviations from HWE were frequent. Three loci (MNCT8, MNZ and MNZZ) were in HWE across all populations and six to eight loci were in HWE in different individual populations (Table 2). Interestingly, only one locus (MNCH) deviated from HWE in all populations. Such non-corresponding distribution of polymorphism in the loci between populations indicates a high degree of isolation between the three islands. We assume that different loci were fixed for one allele or gained mutations in the primer binding sites causing allelic dropout independently in the affected populations.

Five out of 105 locus-pair combinations calculated across all $M$. nesomimi populations of showed moderate deviation from LD $(\mathrm{P}=0.05-0.01)$, but none of the tests were significant after Bonferroni correction. Fst pairwise distances between the populations correspond with the geographic distribution of the host species. The distance between Santa Cruz and Santa Fe populations sharing the same host (Mimus parvulus) was lower $(\mathrm{Fst}=0.179)$ than distances between each of the two islands and the Española population from Mimus macdonaldi (Fst $=0.393$ and 0.214). All Fst values were significant at $\mathrm{P} \leq 0.001$.

In conclusion, we characterised four multiplexed panels of polymorphic microsatellite loci in each of the two louse species and demonstrated their suitability for studies of population history. Low heterozygosity, observed in 
some loci-and-population combinations, points to possible occurrence of null alleles. The impact of inner population subdivision on HWE cannot be fully excluded; but we find the null allele explanation more likely given the distribution of positive LD tests across populations. Samples in each test population were isolated from the same host species and, with the exception of the $P$. serrata Baiersbronn populations showing positive LD tests, only one to two lice per host individual were analysed to avoid inbred or highly related individuals (see Koop et al. 2014). In each species, different loci were usually out of HWE across test populations indicating independent evolution of the populations for many generations. Fst distances between the test populations reflected their respective evolutionary history and/or host origin. Thus the microsatellite loci isolated for $P$. serrata and $M$. nesomimi were shown to provide an appropriate tool for investigation of population substruc- turing and genealogical relationships. These data provide a foundation for further studies of the biogeography and host distribution of these ecologically interesting louse.

Acknowledgements. Research presented in this study was funded by the Czech Science Foundation (project nos. P506/12/P529 and P505/12/1620) and the Marie Curie Fellowship (project Galápagos, no. 235123, FP7-PEOPLE-IEF-2008). V. Roubová was supported by a Team Grant of the Grant Agency of the University of South Bohemia (GAJU, no. 159/2013/P). The material of Polyplax serrata used in the study originates from a louse collection created thanks to a long-term effort of many colleagues from the Laboratory of Molecular Phylogeny and Evolution of Parasites in České Budějovice. We thank to Lukas Keller (University of Zurich) and Paquita Hoeck (Institute for Conservation Research, San Diego) for providing material of Myrsidea nesomimi. The research on M. nesomimi was conducted under permit (no. PC-48-10) from the Galápagos National Park.

\section{REFERENCES}

Ascunce M.S., Toups M.A., Kassu G., Fane J., Scholl K., ReED D.L. 2013: Nuclear genetic diversity in human lice (Pediculus humanus) reveals continental differences and high inbreeding among worldwide populations. PLoS ONE 8: e57619.

Demastes J.W., Spradling T.A., Hafner M.S., Spies G.R., Hafner D.J., Light J.E. 2012: Cophylogeny on a fine scale: Geomydoecus chewing lice and their pocket gopher hosts, Pappogeomys bulleri. J. Parasitol. 98: 262-270.

Fleischer R.C., Loew S., 1995: Construction and screening of microsatellite-enriched genomic libraries. In: J. Ferraris and S. Palumbi (Eds.), Molecular Zoology: Advances, Strategies and Protocols. Wiley-Liss, New York, pp. 459-468.

Hoeck P.E.A., Beaumont M.A., James K.E., Grant R.B., Grant P.R., Keller L.F. 2010: Saving Darwin's muse: evolutionary genetics for the recovery of the Floreana mockingbird. Biol. Lett. 6: 212-215.

Johnson K.P., Clayton D.H. 2004: Untangling coevolutionary history. Syst. Biol. 53: 92-94.

Koblížková A., Doležel J., Macas J. 1998: Subtraction with 3' modified oligonucleotides eliminates amplification artifacts in DNA libraries enriched for microsatellites. Biotechniques 25: 32-34.

Koop J.A., DeMatteo K.E., Parker P.G., Whiteman N.K. 2014: Birds are islands for parasites. Biol. Lett. 10: 20140255.

KRIŠTOFík J., LysÝ J. 1992: Seasonal dynamics of sucking lice (Anoplura) in small mammals (Insectivora, Rodentia) in the natural foci of infections in south-west Slovakia. Biologia 47: 605-617.

Leo N.P., Hughes J.M., Yang X., Poudel S.K.S., Brogdon W.G., BARKER S.C. 2005: The head and body lice of humans are genetically distinct (Insecta: Phthiraptera, Pediculidae): evidence from double infestations. Heredity 95: 34-40.

Light J.E., Smith V.S., Allen J.M., Durden L.A., Reed D.L. 2010: Evolutionary history of mammalian sucking lice (Phthiraptera: Anoplura). BMC Evol. Biol. 10: 292.

Mizukoshi A., Johnson K.P., Yoshizawa K. 2012: Co-phylogeography and morphological evolution of sika deer lice (Damalinia sika) with their hosts (Cervus nippon). Parasitology 139: 1614-1629.

Nessner C.E., Andersen J.J., Renshaw M.A., Giresi M.M., Light J.E. 2014: Characterization of 17 novel polymorphic microsatellite loci in the mammal chewing louse Geomydoecus ewingi (Insecta: Phthiraptera) for population genetic analyses. J. Parasitol. 100: 873-877.

Peakall R., Smouse P.E. 2012: GenAlEx 6.5: genetic analysis in Excel. Population genetic software for teaching and research an update. Bioinformatics 28: 2537-2539.

Peters M.B., Whiteman N.K., Hagen C., Parker P.G., Glenn T.C. 2009: Eight polymorphic microsatellite markers isolated from the widespread avian louse Colpocephalum turbinatum (Phthiraptera: Amblycera: Menoponidae). Mol. Ecol. Resour. 9: 910-912.

Štefka J., Hoeck P.E., Keller L.F., Smith V.S. 2011: A hitchhikers guide to the Galapagos: co-phylogeography of Galapagos mockingbirds and their parasites. BMC Evol. Biol. 11: 284.

ŠTEFKA J., HYPŠA V. 2008: Host specificity and genealogy of the louse Polyplax serrata on field mice, Apodemus species: a case of parasite duplication or colonisation? Int. J. Parasitol. 38: 731-741 